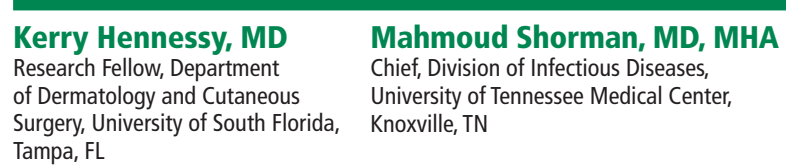

Tampa, FL

\title{
Verrucous plaques on the face, dysphagia, and limb weakness
}

\begin{abstract}
A 59-YEAR-OLD MAN with known chronic obstructive pulmonary disease and poorly controlled diabetes was referred to the hospital by his dermatologist. Two months earlier, he first noticed an itchy "bump" on the cutaneous portion of his upper lip, which then developed into multiple growths that had spread to the tip of his nose.

He reported several episodes of night sweats, dysphagia, and left-sided hand and leg weakness.

He had worked for 30 years in coal mines and sawmills. He was a smoker, with a 25-packyear history. He lived in eastern Tennessee.

Physical examination confirmed numerous exophytic verrucous plaques on his upper cutaneous lip and nose (Figure 1). Results of serum chemistry, liver enzymes, and complete blood cell count were normal. Testing for human immunodeficiency virus infection was negative.

Biopsy of the facial lesions was performed, and he underwent magnetic resonance imaging of the brain and computed tomography of the chest.

Biopsy study showed pseudoepitheliomatous hyperplasia, and Grocott-Gomori methenamine silver staining (Figure 2 ) revealed broad-based budding yeasts measuring 8 to 20 $\mu \mathrm{m}$ in diameter and with thickened cell walls, features consistent with blastomycosis. Because of concern for disseminated disease, the patient was admitted for further workup.
\end{abstract}

\section{FURTHER EVALUATION AND DIAGNOSIS}

Magnetic resonance imaging of the brain revealed ring-enhancing lesions in the pons and inferior cerebellar peduncle. Computed tomography of the chest showed bilateral upperdoi:10.3949/ccjm.87a.19151

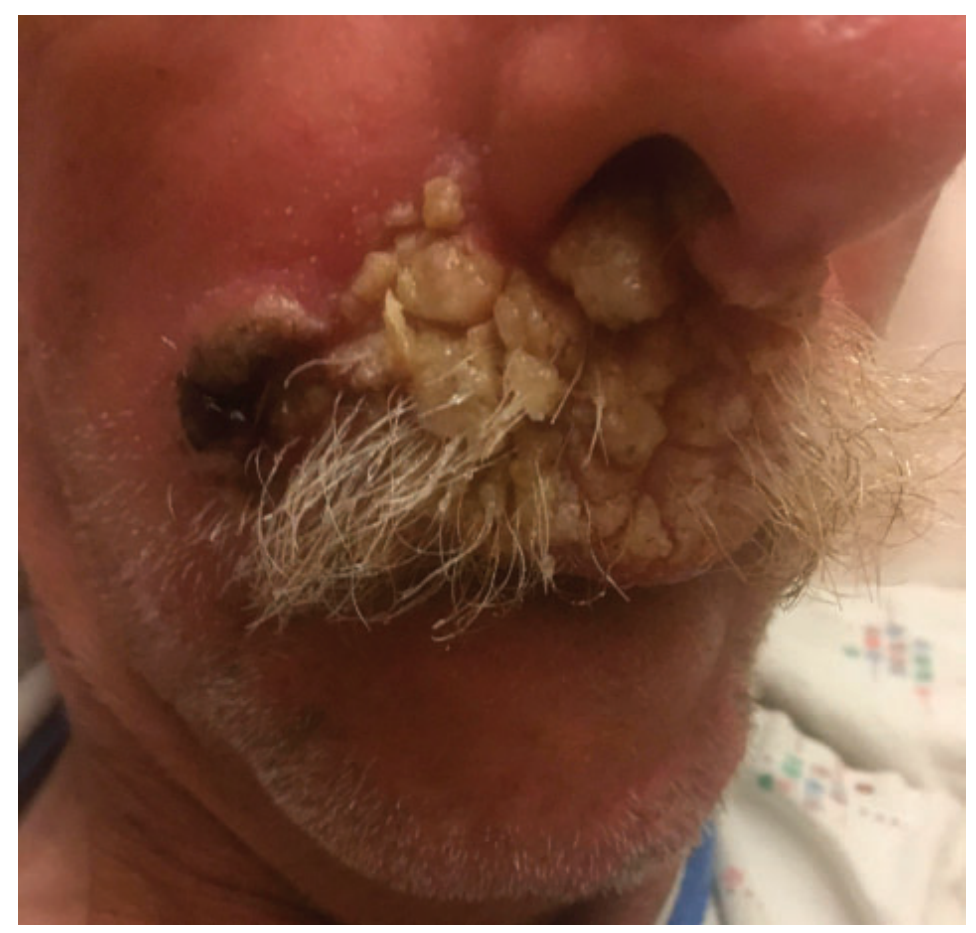

Figure 1. The patient presented with exophytic verrucous plaques on his upper cutaneous lip and nose.

lobe scarring and areas of bronchiectasis, as well as nodules in the upper lobes and groundglass opacities. Urine antigen testing for Blastomyces was positive at $3.07 \mathrm{ng} / \mathrm{mL}$ (reference range for negative, $0 \mathrm{ng} / \mathrm{mL}$ ).

Given these findings and the high likelihood of blastomycosis, sputum cultures and additional serologic testing were not ordered.

The patient was treated with intravenous amphotericin B for 6 weeks and oral voriconazole $200 \mathrm{mg}$ twice daily for 1 year. His skin lesions and neurologic symptoms resolved. Repeat imaging at 4 months showed resolution of brain and chest lesions. 


\section{VERRUCOUS FACIAL PLAQUES}

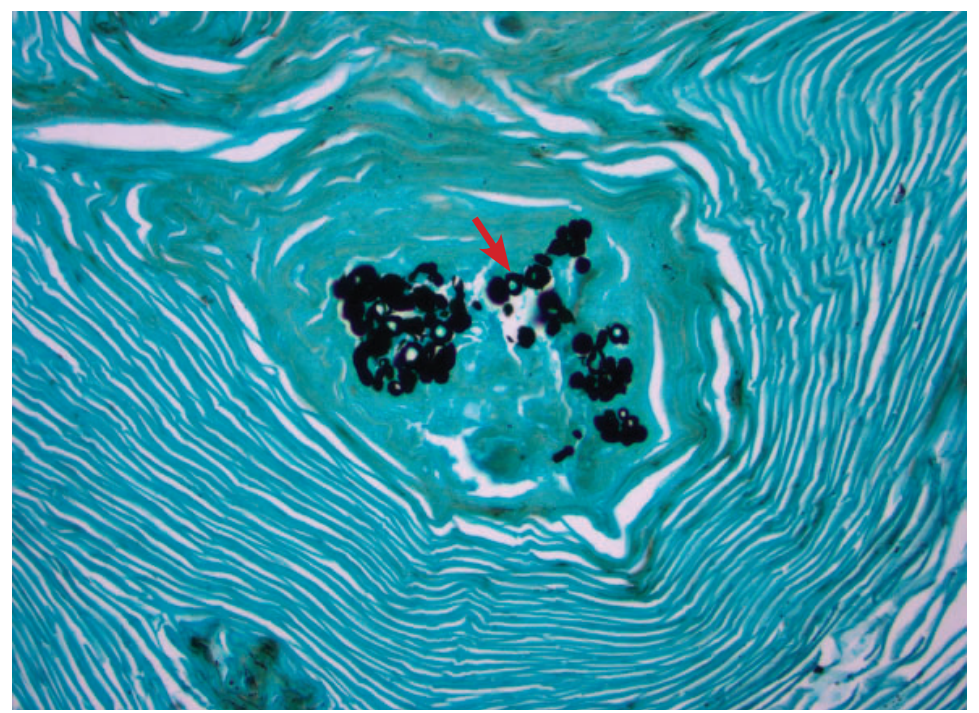

Figure 2. Grocott-Gomori methenamine silver staining revealed broad-based budding yeasts with thickened cell walls (arrow).

\section{BLASTOMYCOSIS: CLUES TO DIAGNOSIS}

The differential diagnosis of cutaneous blastomycosis includes infection with dimorphic fungi (ie, growth forms that change from moldlike to yeastlike), basal cell carcinoma, squamous cell carcinoma, giant keratoacan-
A high index of suspicion in endemic areas aids prompt diagnosis thoma, lupus vulgaris, scrofuloderma, nocardiosis, atypical Mycobacterium infection, syphilis, bromoderma, iododerma, leishmaniasis, granuloma inguinale, lymphoma, and pyoderma gangrenosum. ${ }^{1-3}$

In the diagnosis of dimorphic fungal infections-including blastomycosis, histoplasmosis, and coccidioidomycosis-geographic location, epidemiologic factors, and exposure history are key considerations. Blastomycosis and histoplasmosis are endemic in the Ohio and Mississippi River valleys and southeastern areas of the United States (including eastern Tennessee), whereas coccidioidomycosis is endemic in the southwestern United States. All 3 can present as cutaneous lesions with systemic involvement. Culture and histopathology can help distinguish them.

Basal and squamous cell carcinomas and giant keratoacanthoma can also present as cutaneous plaques. These are easily diagnosed with histopathologic study, and systemic involvement would be uncommon.

Most cases of blastomycosis are considered sporadic. But outbreaks have been associated with recreational and occupational activities involving distribution of soil, including construction, underground exploration, tubing, and hunting. ${ }^{4}$

\section{ROUTES OF TRANSMISSION}

Blastomycosis is transmitted by inhalation of spores and can occur in both immunocompromised and immunocompetent individuals. Large series have shown that most cases of disseminated blastomycosis occur in nonimmunosuppressed individuals. Immunosuppressed patients develop more severe infection and are more likely to have symptomatic dissemination. ${ }^{3}$

$B$ dermatitidis is typically inhaled and affects the lungs, causing pneumonia. However, hematogenous spread has been reported in $25 \%$ to $30 \%$ of cases. ${ }^{5}$ The most common sites for hematogenous spread are the skin, bones, and joints. Rarely, primary skin infection may occur through direct inoculation. ${ }^{6,7}$

In our patient, the presumed source of infection was by inhalation.

Any patient presenting with cutaneous blastomycosis should be investigated for disseminated disease, particularly pulmonary involvement.

\section{DIAGNOSTIC CHALLENGES OF BLASTOMYCOSIS}

Diagnosis of blastomycosis can be challenging, and a high index of suspicion is key in endemic areas.

Culture is the gold standard for diagnosis, but Grocott-Gomori methenamine silver stain can show the presence of broad-based budding yeasts. Unfortunately, multiple biopsies are often necessary for diagnosis, as sensitivities of histology and culture have been reported to be as low as $9.7 \%$ and $61 \%$, respectively. Cultures may take up to 4 weeks for results. ${ }^{8}$

Assays are also available for detection of $B$ dermatitidis antigen in the serum or urine and can yield quicker results. The sensitivity of the urine antigen assay has been reported to be as high as $92.9 \%$, whereas the serum assay is less sensitive. $^{9}$

Cross-reactivity with Histoplasma capsulatum does occur, so a negative result should not preclude a diagnosis of blastomycosis. ${ }^{10}$ 


\section{TREATMENT RECOMMENDATIONS}

Treatment of blastomycosis depends on severity of illness, central nervous system (CNS) involvement, and the patient's immune status. For uncomplicated cases, itraconazole therapy is preferred and is curative in $95 \%$ of cases.

With CNS involvement or life-threatening infection, liposomal amphotericin-B is given. The recommended course is 1 to 2 weeks for severe infection or 6 weeks for CNS involvement. This can then be transitioned to 1 year of oral azole therapy.

Treatment guidelines recommend itraconazole as the preferred agent for all non-

\section{REFERENCES}

1. Bonifaz A, Vazquez-Gonzalez D, Perusquia-Ortiz AM. Endemic systemic mycoses: coccidioidomycosis, histoplasmosis, paracoccidioidomycosis and blastomycosis. German J Dtsch Dermatol Ges 2011; 9(9):705-714. doi:10.1111/j.1610-0387.2011.07731.x

2. Mason AR, Cortes GY, Cook J, Maize JC, Thiers BH. Cutaneous blastomycosis: a diagnostic challenge. Int J Dermatol 2008; 47(8):824830. doi:10.1111/j.1365-4632.2008.03732.x

3. Saccente M, Woods GL. Clinical and laboratory update on blastomycosis. Clin Microbiol Rev 2010; 23(2):367-381. doi:10.1128/CMR.00056-09

4. McBride JA, Gauthier GM, Klein BS. Clinical manifestations and treatment of blastomycosis. Clin Chest Med 2017; 38(3):435-449. doi:10.1016/j.ccm.2017.04.006

5. Miceli A, Krishnamurthy K. Blastomycosis. Accessed June 26, 2020. https://www.ncbi.nlm.nih.gov/books/NBK441987/

6. Gray NA, Baddour LM. Cutaneous inoculation blastomycosis. Clin Infect Dis 2002; 34(10):E44-E49. doi:10.1086/339957

7. Siemieniuk RA, MacFadden DR, Matukas LM. Disseminated blastomycosis with cutaneous involvement in a Canadian railroad worker. Lancet 2015; 385(9971):883. doi:10.1016/S0140-6736(14)61641-X

8. Monaco WE, Batsis JA. A case of disseminated blastomycosis in
CNS disease. Fluconazole and voriconazole are alternatives in patients unable to tolerate itraconazole. ${ }^{11}$ Fluconazole must be given at higher dosages and has higher reported failure rates; however, it has good cerebrospinal fluid penetration. Ultimately, further research must be done to determine the efficacy of various azole agents in step-down treatment of CNS blastomycosis. ${ }^{12,13}$

This patient's presentation highlights the clinical manifestations of disseminated blastomycosis and stresses the importance of maintaining a high index of suspicion in an endemic region.
Vermont. Diagn Microbiol Infect Dis 2013; 75(4):423-425. doi:10.1016/j.diagmicrobio.2013.01.010

9. Durkin M, Witt J, Lemonte A, Wheat B, Connolly P. Antigen assay with the potential to aid in diagnosis of blastomycosis. J Clin Microbiol 2004; 42(10):4873-4875. doi:10.1128/JCM.42.10.4873-4875.2004

10. Bariola JR, Hage CA, Durkin M, et al. Detection of blastomyces dermatitidis antigen in patients with newly diagnosed blastomycosis. Diagn Microbiol Infect Dis 2011; 69(2):187-191. doi:10.1016/j.diagmicrobio.2010.09.015

11. Chapman SW, Dismukes WE, Proia LA, et al; Infectious Diseases Society of America. Clinical practice guidelines for the management of blastomycosis: 2008 update by the Infectious Diseases Society of America. Clin Infect Dis 2008; 46(12):1801-1812. doi:10.1086/588300

12. Bradsher RW Jr. The endemic mimic: blastomycosis an illness often misdiagnosed. Trans Am Clin Climatol Assoc 2014; 125:188-202. pmid:25125734

13. Castillo CG, Kauffman CA, Miceli MH. Blastomycosis. Infect Dis Clin North Am 2016; 30(1):247-264. doi:10.1016/j.idc.2015.10.002

Address: Kerry Hennessy, MD, Department of Dermatology and Cutaneous Surgery, University of South Florida, 13320 USF Laurel Drive, Tampa, Florida 33612; kchennessy@usf.edu 